\title{
Remodeling of Cytokinin Metabolism at Infection Sites of Colletotrichum graminicola on Maize Leaves
}

\author{
Michael Behr, ${ }^{1}$ Václav Motyka, ${ }^{2}$ Fabian Weihmann, ${ }^{1}$ Jiří Malbeck, ${ }^{2}$ Holger B. Deising,${ }^{1,3}$ and \\ Stefan G. R. Wirsel ${ }^{1,3}$ \\ ${ }^{1}$ Institute of Agricultural and Nutritional Sciences, Faculty of Natural Sciences III, Martin-Luther-University Halle-Wittenberg, \\ Betty-Heimann-Str. 3, D-06120 Halle (Saale), Germany; ${ }^{2}$ Institute of Experimental Botany, Academy of Sciences of the Czech \\ Republic, Rozvojová 263, CZ-165 02 Prague 6, Czech Republic; ${ }^{3}$ Interdisziplinäres Zentrum für Nutzpflanzenforschung \\ (IZN), Martin-Luther-Universität Halle-Wittenberg, Betty-Heimann-Str. 3, D-06120 Halle (Saale), Germany
}

Submitted 20 January 2012. Accepted 10 April 2012.

\begin{abstract}
When inoculated onto maize leaves at the onset of senescence, the hemibiotroph Colletotrichum graminicola causes green islands that are surrounded by senescing tissue. Taking advantage of green islands as indicators of sites of the establishment of successful infection and of advanced highperformance liquid chromatography tandem mass spectrometry methodology, we analyzed changes in the patterns and levels of cytokinins (CK) at high spatial and analytical resolution. Twenty individual $\mathrm{CK}$ were detected in green islands. Levels of cis-zeatin-9-riboside and cis-zeatin-9riboside-5'-monophosphate increased drastically, whereas that of the most prominent $\mathrm{CK}$, cis-zeatin- $O$-glucoside, decreased. The fungus likely performed these conversions because corresponding activities were also detected in in vitro cultures amended with $\mathrm{CK}$. We found no evidence that $\boldsymbol{C}$. graminicola is able to synthesize CK entirely de novo in minimal medium but, after adding dimethylallyl diphosphate, a precursor of CK biosynthesis occurring in plants, a series of trans-zeatin isoforms (i.e., trans-zeatin-9-riboside5'-monophosphate, trans-zeatin-9-riboside, and trans-zeatin) was formed. After applying CK onto uninfected leaves, transcripts of marker genes for senescence, photosynthesis, and assimilate distribution were measured by quantitative reverse-transcribed polymerase chain reaction; furthermore, pulse-amplitude modulation chlorophyll fluorometry and single-photon avalanche diode analyses were conducted. These experiments suggested that modulation of CK metabolism at the infection site affects host physiology.
\end{abstract}

Cytokinins (CK) control crucial aspects of plant development such as postembryonic organogenesis (Pernisova et al. 2009); shoot, root (Kyozuka 2007), and cambial meristem activities (Matsumoto-Kitano et al. 2008); and senescence (Buchanan-Wollaston 1997; Kim et al. 2006). Evidence has accumulated that $\mathrm{CK}$ are produced not only in roots but also in aerial organs (Sakakibara 2006). CK constitute a structurally complex group of compounds derived from adenine that can carry diverse isoprenoid or aromatic side chains at the $N^{6}$ position (Mok and Mok 2001; Sakakibara 2006). Isoprenoid CK

\section{Behr and V. Motyka contributed equally to this work.}

Corresponding author: S. G. R. Wirsel; Telephone: +49 (0)345 5522672; Fax: +49(0)345 5527120; E-mail: stefan.wirsel@landw.uni-halle.de

* The $e$-Xtra logo stands for "electronic extra" and indicates that two supplementary tables and one supplementary figure are published online. are usually categorized according to their structure and physiological activity into four functionally different groups, including i) bioactive nucleobases and ribosides (iP, iPR, Z, ZR, cisZ, cisZR, DHZ, and DHZR) (for structures and abbreviations see Supplementary Table S1), ii) inactive or weakly active $N$-glucosides (iP7G, iP9G, Z7G, Z9G, cisZ7G, cisZ9G, DHZ7G, and DHZ9G), iii) inactive or weakly active $O$-glucosides (ZOG, ZROG, cisZOG, cisZROG, DHZOG, and DHZROG), and iv) nucleotides representing the first products of CK biosynthesis (iPRMP, ZRMP, cisZRMP, and DHZRMP). Conventionally, the nucleobases are known as the active forms of CK that are recognized by membrane-bound receptors to mediate intracellular signaling (Kieber and Schaller 2010). Ribosylated forms (nucleosides) are considered transport forms of $\mathrm{CK}$, whereas ribosylphosphates (nucleotides) may constitute a reservoir of precursors for potential side-chain modifications. The $O$-glucosides of CK are regarded as storage forms that can be reversibly converted into their corresponding nucleobases (Frebort et al. 2011; Letham and Palni 1983; Mok and Mok 2001). $\mathrm{N}$-glucosylation of CK is irreversible, rendering such forms probably irretrievably inactivated (Frebort et al. 2011; Letham and Palni 1983; Mok and Mok 2001).

The most typical representative of isoprenoid $\mathrm{CK}$ and the most active in a number of bioassays is zeatin, which occurs as two isomers, cis and trans, referring to the position of the terminal hydroxyl group at the isoprenoid side chain. In the past, cis-zeatin (cisZ) and its derivatives were largely overlooked, being considered as adjunct to $\mathrm{CK}$ homeostasis, with only weak biological impact. The relevance of cisZ-type CK has just recently begun to emerge, due to the impressive progress in $\mathrm{CK}$ analysis, based on ultra-performance liquid chromatography (UPLC) in combination with sensitive and selective detection (tandem mass spectrometry [MS/MS]) (Kojima et al. 2009; Novak et al. 2008). The cisZ-type CK occur in high concentrations exceeding those of corresponding trans $\mathrm{Z}(\mathrm{Z})$ types in many plants. In a comprehensive screening of over 150 land plants, cisZ has been found to be ubiquitous throughout the plant kingdom. In many monocotyledons, including maize, cisZ-type CK accounted for approximately $90 \%$ of the total $\mathrm{CK}$ pool, with $\mathrm{cisZ}$ - $\mathrm{O}$-glucoside (cisZOG) and its 9-riboside (cisZROG) being the most prevalent (Gajdosova et al. 2011). Although it is still not clear whether cisZ plays a critical role in plant development, the distinct isoprenoid origins of $\mathrm{Z}$ and cisZ (Kasahara et al. 2004) suggest that plants are able to modulate independently, individually, and specifically the levels of these CK isoforms. In addition to cis-trans isomerization, further consequences of structural CK diversity for plant physiol- 
ogy are just beginning to emerge, including the role of $\mathrm{CK}$ diand triphosphates as biosynthetic precursors; interconversion of free bases, ribosides, and nucleotides; side-chain hydroxylation; and reduction (Sakakibara 2006).

Beyond their significance for plant development, CK are also involved in pathogenic and symbiotic interactions between plants and microbes, including bacteria (Costacurta and Vanderleyden 1995; Frugier et al. 2008; Pertry et al. 2010; Sakakibara et al. 2005), protista (Ludwig-Müller and Schuller 2008), and fungi (Barker and Tagu 2000; Vadassery et al. 2008). It has been proposed that $\mathrm{CK}$ may play a role in the interactions of plants with biotrophic fungal pathogens (i.e., rusts and mildews) (Ashby 2000). In the latter pathosystems, particular symptoms called green islands occur. It is unknown whether biotrophic fungi either synthesize and actively secrete $\mathrm{CK}$ at infection sites or stimulate the host to increase CK levels, leading to locally increased invertase activity and creation

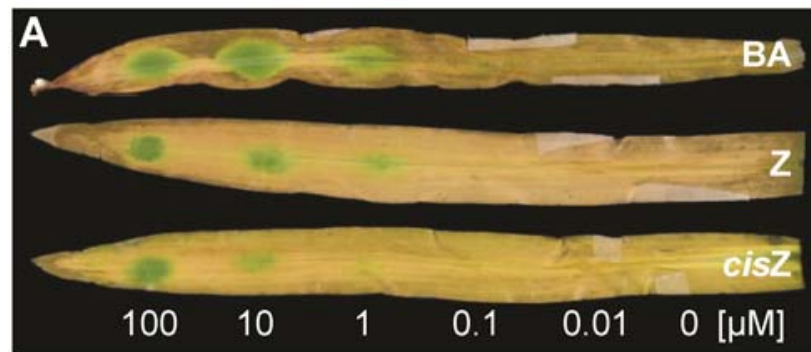

B
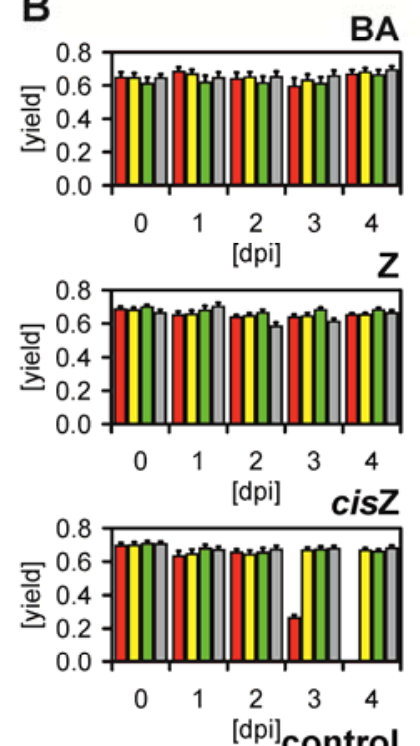

C

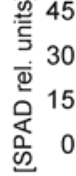

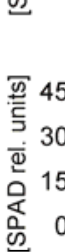
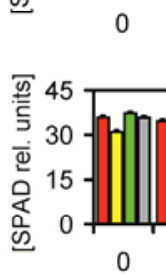

1

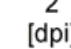

2

cisZ

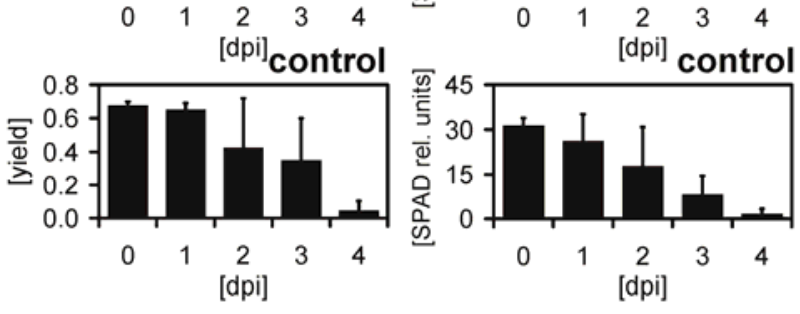

$0 \mu \mathrm{M} \quad \square 0.1 \mu \mathrm{M} \quad \square 1 \mu \mathrm{M} \quad \square 10 \mu \mathrm{M} \quad \square 100 \mu \mathrm{M}$

Fig. 1. Effects of cytokinin (CK) treatment on senescence, photosynthesis, and chlorophyll content of maize. A, Treatment of detached senescing leaves with $N^{6}$-benzyladenine (BA), trans-zeatin (Z), and cis-zeatin (cisZ) at the indicated concentrations. Photograph was taken at 3 days postinoculation; B, and $\mathbf{C}$, 28-day-old whole plants were sprayed with BA, Z, and cis $\mathrm{Z}$ at the indicated concentrations. The still-attached third leaves were used to determine photosynthetic yield by $\mathbf{B}$, pulse-amplitude modulation chlorophyll fluorometry and $\mathbf{C}$, chlorophyll content by single-photon avalanche diode analysis. of a carbohydrate sink, the delay of senescence, and the appearance of green islands (Walters and McRoberts 2006). In order to understand the impact of alterations in CK levels and composition on pathogenicity, analyses have to be performed at high spatial resolution (i.e., exactly at the sites of infection), at which CK-mediated modulation of plant metabolism may occur. However, experimental proof for the impact of fungal pathogens on $\mathrm{CK}$ profiles at the infection site is scarce because previous investigations were carried out with extracts from whole, infected plant organs. Because infection sites represent only a minor part of the total leaf tissue at early stages of pathogenesis, infection-mediated changes in the CK profiles observed thus far may reflect only a part of the alterations.

We have previously shown that green islands occur also in the compatible interaction of Zea mays with the hemibiotrophic ascomycete Colletotrichum graminicola (Behr et al. 2010). In this pathosystem, green islands develop only on infected leaves at early senescence, whereas on mature leaves, a different symptom appears, which is called anthracnose. Although the fungus causes extended tissue collapse in anthracnose lesions, only little damage to the host plant occurs in green islands during initial stages of pathogenesis. Furthermore, in green islands, the fungal infection delays the decline of photosynthetic activity in a highly localized fashion (i.e., exactly at the infection site). Quantitative reverse-transcribed polymerase chain reaction (qRT-PCR) indicated that the transcript levels of the incwl gene, encoding a maize cell-wallbound invertase, were drastically increased in green islands. We reasoned that increased invertase activity induced a local carbon sink supporting fungal growth during the biotrophic phase of pathogenesis (Behr et al. 2010). In combination with specific access to green islands (Behr et al. 2010), recent improvements of analytical methods (Dobrev and Kaminek 2002; Hoyerova et al. 2006; Kojima et al. 2009; Novak et al. 2008) have made it possible to detect and quantify a considerably increased number of $\mathrm{CK}$ than in previous studies.

In this article, we combine precise tissue excision with highresolution $\mathrm{CK}$ analyses to investigate the entire $\mathrm{CK}$ profile and quantify the amounts and changes of individual $\mathrm{CK}$ at fungal infection sites in green islands. Furthermore, we examine the capability of $C$. graminicola to convert exogenously applied $\mathrm{CK}$ in vitro and assess the capability of the fungus to synthesize CK de novo.

\section{RESULTS}

CK mimic fungal activity in delaying senescence and the decline of photosynthesis.

The hemibiotroph $C$. graminicola is able to delay senescence of host leaves at infection sites, resulting in appearance of green islands. We applied small droplets of Z, cisZ, and $N^{6}$ benzyladenine (BA) solutions onto detached and still green maize leaves. All applied CK delayed senescence, as made evident by the maintenance of green spots at the sites of application. The green spots were surrounded by yellow senescing leaf tissue that had developed during 3 days of incubation (Fig. 1A). BA appeared to be the most effective CK, followed by $Z$ and cisZ. Leaves that were already yellow at the time of treatment did not show re-greening with any of the CK at any concentration tested (not shown).

We used pulse-amplitude modulation (PAM) chlorophyll fluorometry and single-photon avalanche diode (SPAD) analyses to determine whether the apparent delay of senescence corresponded to altered photosynthetic activity and chlorophyll contents, respectively. Leaves from untreated controls that had reached the early stages of senescence at the beginning of the experiment had lost photosynthetic activity within 4 days (Fig. 
1B). Application of Z, cisZ, and BA delayed or completely abolished this process. Correspondingly, SPAD analyses showed that treatment with each of these compounds resulted in a slower decline of the relative chlorophyll contents (Fig. 1C). These results indicated that, in maize, $\mathrm{CK}$ are sufficient to cause occurrence of green islands and to delay the decay of photosynthetic activity and the degradation of chlorophyll during leaf senescence. In this regard, $\mathrm{Z}$ appeared more effective than cisZ.

\section{Effects of CK on expression of markers}

of photosynthesis, senescence, and assimilate distribution.

We had previously employed qRT-PCR assays for maize genes encoding invertases (incw 1 to incw 4 , ivrl, and ivr2), and marker proteins for senescence (seel, see $2 b$, and ccpl encoding proteases) and photosynthesis (me1, pep1, cp29, RbcS1, and $\mathrm{RbcS} 2$ encoding the C4-specific isoforms of malic enzyme and phosphoenolpyruvate carboxylase, chlorophyll a/b-binding protein, and two isoforms of the small subunit of ribulose1,5-biphosphate carboxylase, respectively) (Behr et al. 2010). Fungal infection caused specific transcriptional responses of some of these genes, most notably of incwl, encoding a cell wall invertase. Here, we investigated whether a treatment of whole plants with $\mathrm{Z}$ or cis $\mathrm{Z}$ may evoke similar transcriptional responses of these genes. For this purpose, leaf number 3, which was at an early stage of senescence when sprayed, and leaf number 5 , which was mature, were analyzed. The transcript levels of see 1 and see $2 b$ were slightly increased by Z and cis $\mathrm{Z}$ on leaf number 3 , and those of ccpl only by cis $\mathrm{Z}$ (Supplementary Fig. S 1). These effects vanished on leaf number 5 . Treatment with both $\mathrm{CK}$ resulted in reduced transcript abundances of the photosynthesis marker genes, except for pep1 and cp29 on leaf number 3. Among the genes encoding invertases, the transcript levels of incwl were increased on leaf number 3 after CK treatment. Transcript abundances of the other invertase genes either showed no distinct response to $\mathrm{CK}$ application or decreased.

\section{C. graminicola infection locally changes CK profiles.}

We quantified CK levels at a spatial resolution comparable with that previously attained in qRT-PCR assays analyzing transcript levels at infection sites (Behr et al. 2010). Extracts obtained from tissue samples excised from infected leaf number 3 , representing green islands and the area surrounding them, were analyzed by high-performance liquid chromatography (HPLC) MS/MS and were compared with those from a noninfected control leaf of the same age. In general, the $O$-glucosides constituted the majority of the total CK contents, whereas the nucleobases and the nucleosides were found at lower concentrations (Fig. 2A). The nucleotides and $\mathrm{N}$-glucosides occurred only in trace amounts. In the noninfected leaf number 3 , the levels of cis-isomers of zeatin-type CK considerably exceeded those of the corresponding trans-isomers (Fig. 2B; Supplementary Table S2), confirming previous data reported for maize (Gajdosova et al. 2011; Veach et al. 2003). The most prevalent CK derivative was cisZOG, representing $89 \%$ of total CK. Infection caused a decrease of total CK contents of $46 \pm 4 \%$ in green islands and $33 \pm 11 \%$ in tissue surrounding such infection sites (Fig. 2A). This likely resulted from considerably diminished levels of $O$-glucosides whereas the nucleobases remained relatively constant. The nucleosides and nucleotides were also increased, especially those derived from cisZ. For some individual CK derivatives, drastic changes were observed (Fig. 2B). The cisZ-9riboside (cisZR) and cisZ-9-riboside-5'-monophosphate (cisZRMP) contents increased substantially by $733 \pm 5$ and $1,660 \pm 25 \%$, respectively, at green islands compared with the noninfected control. Conversely, the cisZOG content decreased by $54 \pm 6 \%$. Evidently, for some CK (e.g., cisZR), clear differences existed between tissue sampled from green islands and the surrounding tissue, indicating an infection-site-directed concentration gradient over a distance of up to $5 \mathrm{~mm}$.
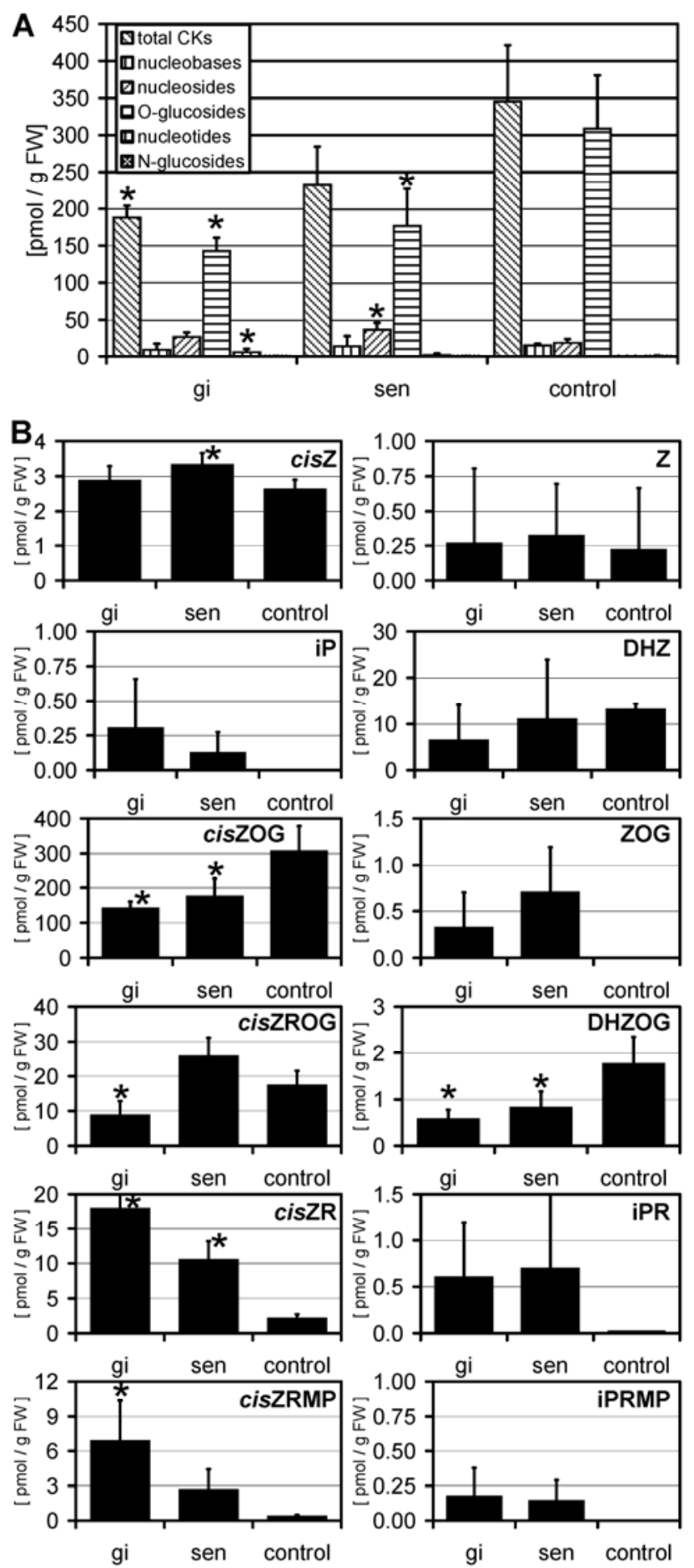

Fig. 2. Qualitative and quantitative cytokinin (CK) determination at fungal infection sites. CK analysis by high-performance liquid chromatography tandem mass spectrometry used extracts obtained from number 3 leaves harvested at 4 days postinoculation that were either untreated (control) or inoculated with Colletotrichum graminicola (gi, sen). Infected leaves were analyzed by using excised tissue samples originating either from infection sites visible as green islands (gi) or the noninfected senescent tissues surrounding them (sen). A, CK combined in subgroups. B, Individual CK showing concentrations altered after fungal infection. Asterisks on top of the columns indicate significant differences between gi and sen when compared with the corresponding controls. 
CK metabolism of $C$. graminicola in vitro.

The above results suggested a conversion of certain CK at fungal infection sites by either host or fungal enzymes or both. To determine the capability of $C$. graminicola to alter CK profiles at the infection site, we took three approaches to analyze whether or not $C$. graminicola is able to convert or de novo synthesize $\mathrm{CK}$ in fungal in vitro cultures. In the first approach, we used a cell-free extract prepared from maize leaves to expose the fungus to a naturally occurring mixture of $\mathrm{CK}$, similar to what the fungus encounters during pathogenesis. In total, six variants were analyzed by HPLC MS/MS, three using extracts from mature green leaves and three using extracts from yellow, senescing leaves. As expected, CK contents were generally higher in the former (Fig. 3A). For each of the two extracts (i.e., from both green and yellow leaves), in two of the
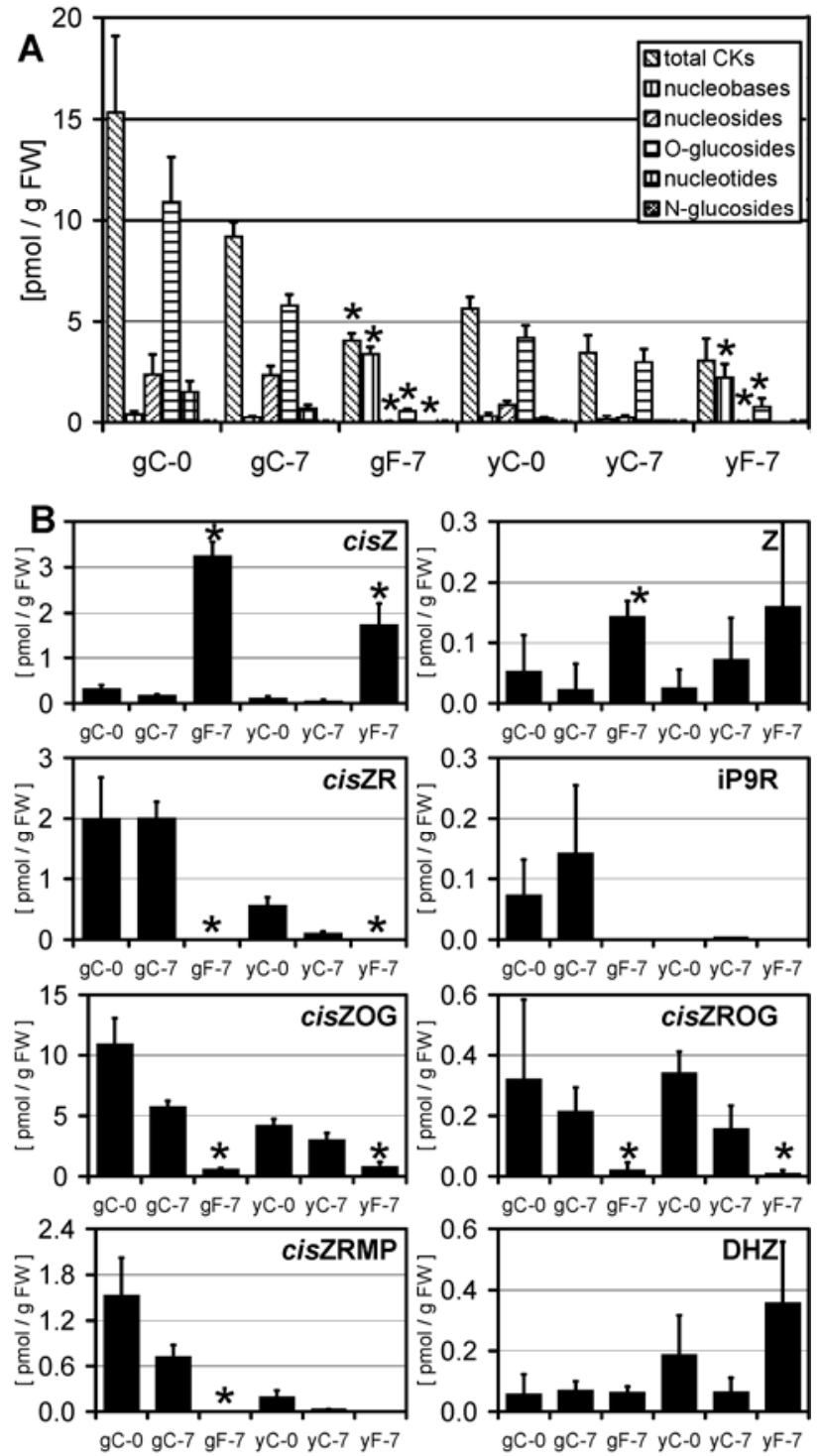

Fig. 3. In vitro conversion of maize-derived cytokinins (CK) by Colletotrichum graminicola. Media contained extracts of maize leaves that were young and green $(\mathrm{g})$ or senescing and yellow $(\mathrm{y})$ when harvested for preparation. $\mathrm{C}-0$ and $\mathrm{C}-7=$ noninoculated controls at the beginning and after 7 days, respectively; F-7 = culture of $C$. graminicola after 7 days of incubation. CK analysis by high-performance liquid chromatography tandem mass spectrometry used the supernatants. A, CK combined in subgroups. B, Individual CK showing concentrations altered by fungal activity. Asterisks on top of the columns indicate significant differences for $\mathrm{gF}-7$ and $\mathrm{yF}-7$ (supernatants of fungal cultures at 7 days) when compared with the corresponding noninoculated controls ( $\mathrm{gC}-7$ and $\mathrm{yC}-7)$. three treatments, $\mathrm{CK}$ were analyzed in noninoculated media at the beginning ( 0 days postinoculation [dpi]) and at the end ( 7 dpi) of the incubation period. These noninoculated controls showed to which extent CK decomposed under the experimental conditions used. Both media (i.e., those made from green as well as those from yellow leaves) showed some decline for total $\mathrm{CK}$ contents during an incubation period of 7 days (Fig. 3A). In the third treatment, both extracts (i.e., from green as well as yellow leaves) were inoculated with $C$. graminicola and analyzed after 7 days. Here, fungal metabolic activity led to an additional reduction of total $\mathrm{CK}$ contents. These decreases amounted to $56 \pm 4 \%$ in the medium prepared from green leaves and to $11 \pm 17 \%$ in the medium from senescing leaves, as compared with the corresponding noninoculated controls measured at day 7 . In marked contrast, the contents of $\mathrm{CK}$ nucleobases, representing the active forms of $\mathrm{CK}$, were significantly increased by the fungus (Fig. 3B). This effect was strongest for cisZ, which increased by $1,744 \pm 5$ and $3,867 \pm$ $13 \%$ in extracts prepared from green and yellow leaves, respectively, compared with the corresponding noninoculated controls. These increases were accompanied by strong decreases in some of the CK $O$-glucosides, nucleosides, and nucleotides, suggesting that $\mathrm{CK} O$-glucosides were transformed to the corresponding nucleobase. Interestingly, most of these changes were again associated with the cis-isoforms (i.e., cisZOG, cisZR, and cisZRMP).

In the second approach, we used minimal medium that was amended with individual commercially available $\mathrm{CK}(\mathrm{Z}$, cisZ, trans-zeatin-9-riboside [ZR], cisZR, trans-zeatin-9-riboside$5^{\prime}$-monophosphate [ZRMP], and cisZRMP) to test whether $C$. graminicola is able to convert these $\mathrm{CK}$ in vitro and, if so, which products are formed. HPLC MS/MS analyses were performed for each of these $\mathrm{CK}$ in the presence or absence of the fungus, and before and 7 days after inoculation (Fig. 4; the first designation of $\mathrm{CK}$ on the top of each box refers to the amended $\mathrm{CK}$, the second indicates the $\mathrm{CK}$ measured). In addition, CK profiles were analyzed in the supernatants and in the mycelia of fungal cultures incubated for 7 days. Because the commercially available cisZRMP was heavily contaminated by cisZR (data not shown), it was not further considered in these investigations. All other supplemented $\mathrm{CK}$ remained stable during the period of the experiment of 7 days (Fig. 4). However, in all supernatants of fungal cultures, the concentration of the supplemented CK (i.e., Z, cisZ, ZR, cisZR, and ZRMP) had decreased, suggesting that $C$. graminicola had metabolized these compounds to some extent. The reduction of the concentration of supplemented CK was accompanied by the increase of other $\mathrm{CK}$ derivatives in the supernatant or mycelium. Amended cis $\mathrm{Z}$ was efficiently isomerized to $\mathrm{Z}$ in the mycelium, whereas $\mathrm{Z}$ was partially taken up into the mycelium but only poorly isomerized to cisZ. Amended ZR was transformed to various other $\mathrm{CK}$, because $\mathrm{Z}$ and cisZRMP were identified in the supernatant, and cisZ, cisZR, and even dihydrozeatin-9-riboside (DHZR) in the mycelium. Addition of cisZR to the media was associated with accumulation of miscellaneous CK derivatives, including cisZ, cisZRMP, and especially, ZRMP in the supernatant and of $Z$ and, to a much lower extent, cisZ in the mycelium. Supplemented ZRMP appeared to be transformed to $\mathrm{Z}$ and $\mathrm{ZR}$ as measured in the supernatant.

In addition, for one of the amended compounds, ZRMP, we recorded a time series to gain deeper insight into the succession of conversions (Fig. 5). Its concentration decreased steadily over a period of 12 days, whereas ZR was detected as an intermediate product peaking at 3 days that was further converted to $Z$ with a maximal level at 7 days. The observed sequence of metabolic steps suggests that ZRMP was first dephosphorylated and then deribosylated to yield Z. Small 
amounts of cisZOG were detected after ZRMP application during the whole incubation period (data not shown).

The third approach assessed the ability of $C$. graminicola to synthesize CK de novo in minimal medium. CK were not detectable above background levels after 7 days of growth in unamended Czapek-Dox minimal medium (data not shown). Therefore, we supplemented the medium with putative substrates (dimethylallyl diphosphate [DMAPP] with AMP, ADP, and ATP) of CK biosynthetic enzymes (i.e., isopentenyltransferases) (Kakimoto 2001; Sakakibara 2006), to test whether the fungus might utilize these substances to synthesize CK. HPLC MS/MS measurements were carried out for control media lacking the fungus and for inoculated media; in case of the latter, immediately and at 3 dpi (Fig. 6). Analysis of the medium immediately after inoculation showed that conidia did not contribute to $\mathrm{CK}$ accumulation. Analysis of mycelium at 3 dpi revealed substantial amounts of Z, ZR, and ZRMP, indicating that synthesis has occurred. Also, other CK such as cisZ, cisZRMP, dihydrozeatin (DHZ), and DHZR occurred in the mycelium at $3 \mathrm{dpi}$; however, at considerably lower levels compared with $\mathrm{Z}$ derivatives. The supernatant contained minor levels of CK.

In summary, the three approaches using in vitro cultures indicate that $C$. graminicola has the capacity to interconvert certain $\mathrm{CK}$ occurring naturally in maize and, furthermore, to produce CK using DMAPP as a precursor.

\section{DISCUSSION}

Infection of senescing maize leaves by the hemibiotroph $C$. graminicola evokes the formation of green islands. We show here that the application alone of individual CK onto senescing leaves caused physiological responses that corresponded to those observable after fungal infection. Quantification of CK levels exactly at fungal infection sites suggests that storage $\mathrm{CK}$ ( $O$-glucosides) are locally converted to other CK forms. $C$. graminicola has the capacity to perform several CK interconversions in the absence of the host plant in in vitro cultures and, furthermore, to produce bioactive $\mathrm{CK}$ if the growth medium had been exogenously amended with precursors of $\mathrm{CK}$ biosynthesis.

Several physiological responses of maize have previously been noticed to occur at green islands during the infection with C. graminicola (Behr et al. 2010). Most of these responses are also seen after treatment of maize leaves at early stages of senescence with CK. Although all CK tested in this study were biologically active with respect to maintenance of photosynthetic activity and chlorophyll contents as determined by PAM and SPAD analyses, the impact of $Z$ was stronger than that of cisZ. This corresponds to a generally reported lower activity of cis $\mathrm{Z}$ types compared with $\mathrm{Z}$ forms in a number of $\mathrm{CK}$ bioassays (Gajdosova et al. 2011; Kaminek et al. 1979). Although cis-trans isomerases of CK exist in plants (Bassil et al. 1993), isomerization of the exogenously applied CK was not found at such a degree in maize leaves that differences between $\mathrm{Z}$ and cisZ disappeared.

Both fungal infection and treatment with $\mathrm{Z}$ enhanced transcript levels of incwl on senescing leaves. This particular cellwall-bound invertase gene exhibited the strongest transcriptional stimulation among 16 maize genes analyzed at green islands during the infection of senescing leaves with $C$. graminicola (Behr et al. 2010). Cell-wall-bound invertase is a key enzyme determining sink strength, and the activity of these enzymes or transcript levels of the corresponding genes have been shown to rise upon infection in some pathosystems (Wingler and Roitsch 2008). A link between invertase and CK has previously been shown in transgenic tobacco, in which
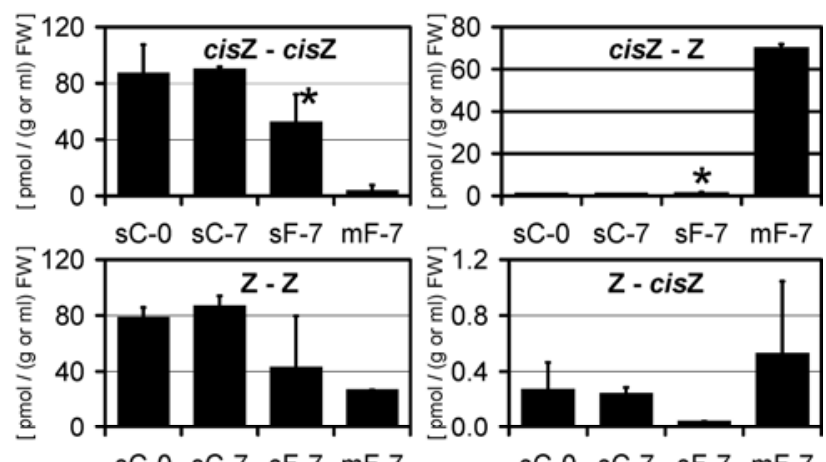

$\begin{array}{llll}\mathrm{sC}-0 & \mathrm{sC}-7 & \mathrm{sF}-7 & \mathrm{mF}-7\end{array}$

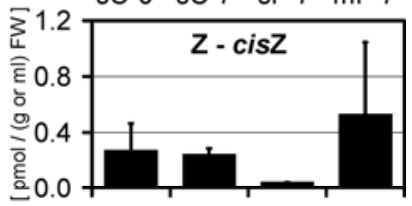

sC-0 sC-7 sF-7 mF-7

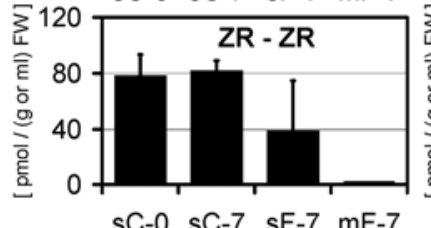

sC-0 sC-7 $\quad$ sF-7 $\quad \mathrm{mF}-7$
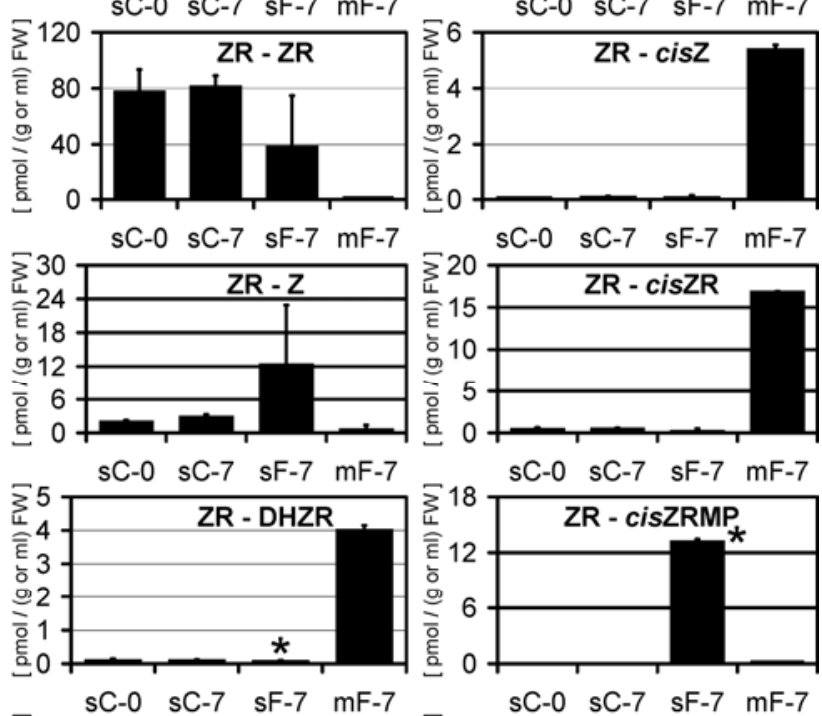

sC-0 sC-7 sF-7 $\quad \mathrm{mF}-7$

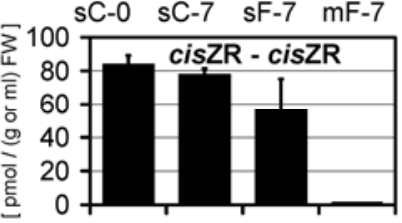

sC-0 sC-7 sF-7 mF-7
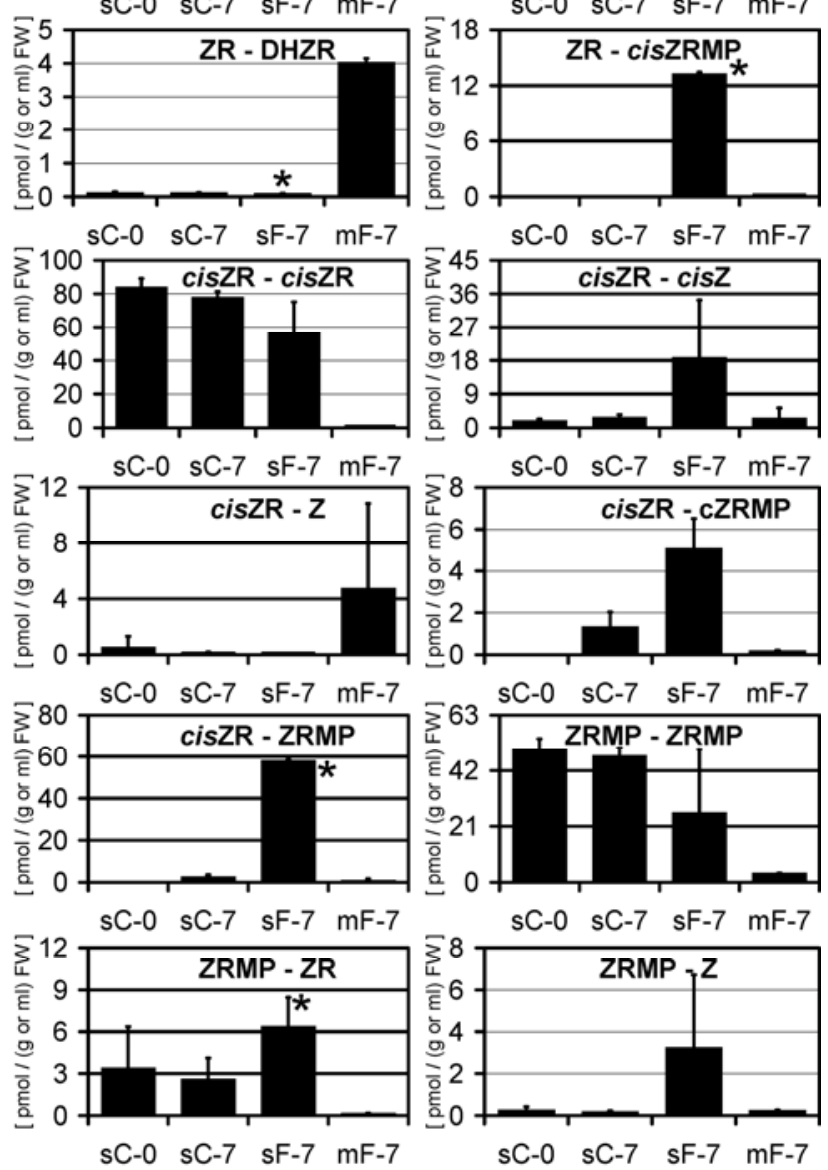

Fig. 4. In vitro conversion of individual cytokinins (CK) by Colletotrichum graminicola. The first designation of CK shown on top of each box refers to the $\mathrm{CK}$ added to the particular minimal medium, the second indicates the measured CK as determined by high-performance liquid chromatography tandem mass spectrometry. In all cases, measurements are first demonstrated for the particular CK added and further for CK derivatives showing alterations. Labels on $x$ axis read as follows: $\mathrm{s}$ and $\mathrm{m}, \mathrm{CK}$ analysis using the supernatant and the mycelium, respectively; $\mathrm{C}$, noninoculated control; $\mathrm{F}$, fungal culture; -0 and -7 , analysis at the beginning and after 7 days, respectively. Asterisks indicate significant differences between sF-7 (supernatant of fungal culture at 7 days) and the corresponding noninoculated control (sC-7). 
endogenously increased CK levels caused an increase in extracellular invertase activity, resulting in a delay of senescence (Balibrea Lara et al. 2004). Here, we provide evidence that such a link could be also important for the interaction of $C$. graminicola with maize, supporting the hypothesis that, at infection sites, CK stimulate a signaling cascade leading to an enhanced host invertase activity (Walters and McRoberts 2006).

Our measurements of $\mathrm{CK}$ at green islands demonstrate that, indeed, fungal infection leads to local changes in the concentrations of certain CK. Notably, the storage CK cisZOG, which reaches the highest concentration of all individual $\mathrm{CK}$ in the noninfected maize leaf, decreased considerably after infection.
Concomitantly, the levels of cisZR and cisZRMP increased. Because concentration gradients exist for these three $\mathrm{CK}$, the respective effect is stronger at green islands compared with the surrounding senescing tissue, indicating that $\mathrm{CK}$ metabolism is primarily affected at the infection site.

CK levels were measured previously in other pathosystems. However, in these experiments, whole infected plant organs were used, with lower analytical and spatial resolution. These experiments suggested that total amounts of CK increased in green islands evoked by biotrophs (Walters and McRoberts 2006). In contrast, our analyses found decreased total CK levels. It needs to be considered that our measurements allowed the detection of more individual CK compounds, particularly

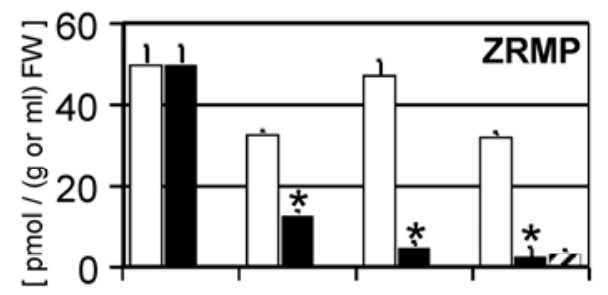

$0 d \quad 3 d \quad 7 d \quad 12 d$

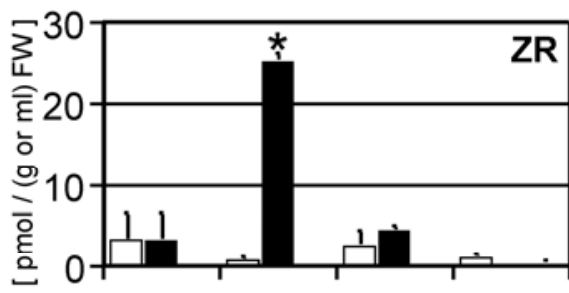

$0 \mathrm{~d} \quad 3 \mathrm{~d} \quad 7 \mathrm{~d} \quad 12 \mathrm{~d}$

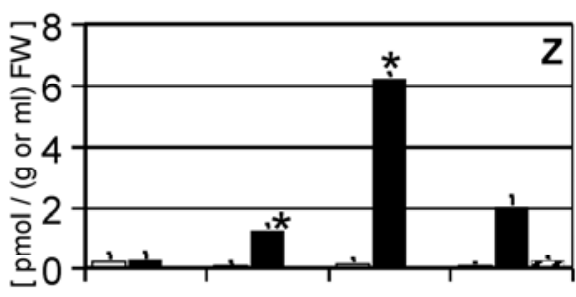

$0 d \quad 3 d \quad 7 d$

$12 \mathrm{~d}$

mycelium

Fig. 5. Time course of in vitro conversion of trans-zeatin-9-riboside-5'-monophosphate (ZRMP) by Colletotrichum graminicola. Data show the results of high-performance liquid chromatography tandem mass spectrometry analyses of the amended cytokinin (CK) ZRMP and its metabolites trans-zeatin-9-riboside (ZR) and trans-zeatin (Z), whose concentrations considerably changed during incubation. A noninoculated control documented putative decomposition. CK were analyzed in the supernatants at the beginning ( 0 days) and during incubation $(3,7$, and 12 days). At the end, CK were also measured in the harvested mycelium. Asterisks indicate significant differences between the supernatant of the fungal culture and the corresponding noninoculated control.
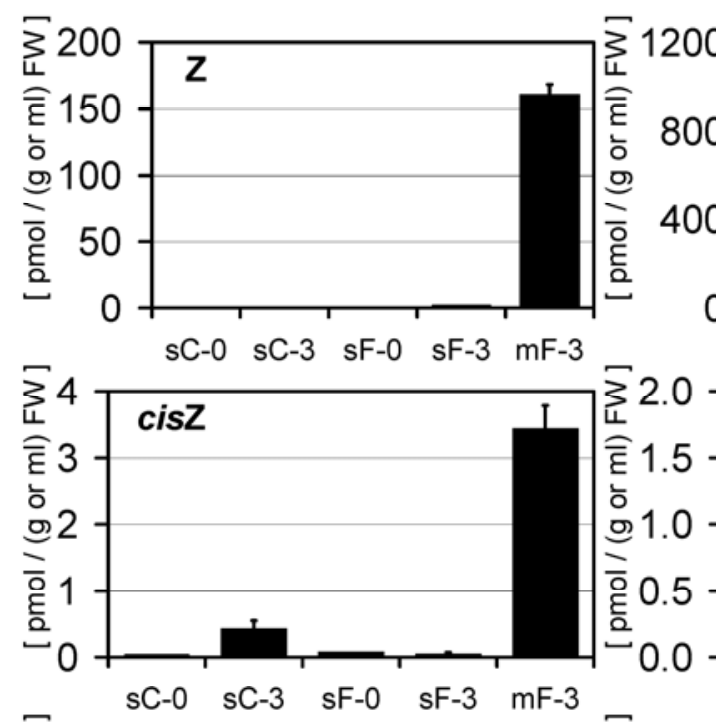

0
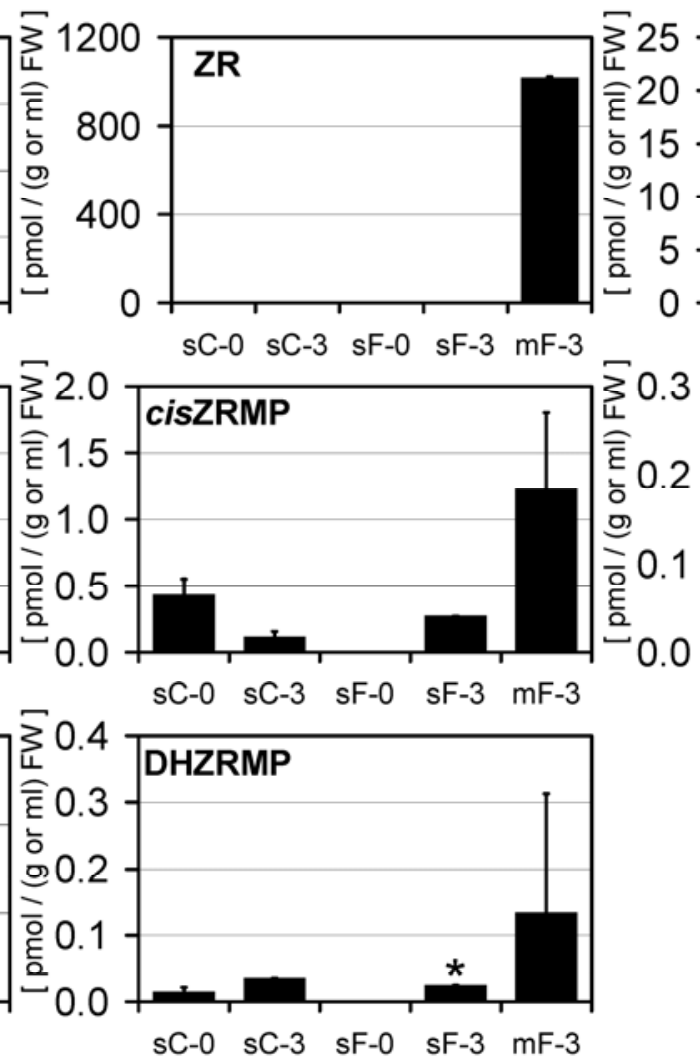

DHZRMP

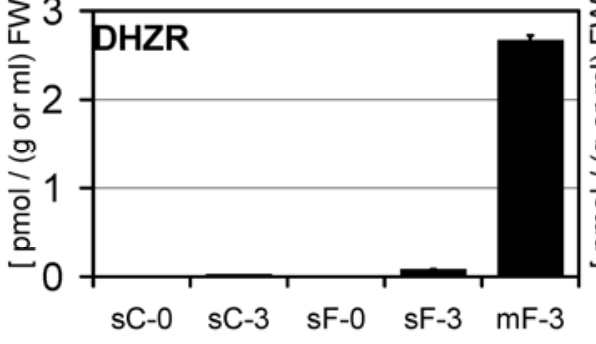

Fig. 6. In vitro synthesis of cytokinins (CK) by Colletotrichum graminicola. Minimal medium was amended with a mixture of dimethylallyl diphosphate (DMAPP), with AMP, ADP, and ATP (100 nM each). Labels on $x$ axis read as follows: $\mathrm{s}$ and m, CK analysis using the supernatant and the mycelium, respectively; C, noninoculated control; F, fungal culture; -0 and -3 , analysis at the beginning and after 3 days, respectively. Data show the results of high-performance liquid chromatography tandem mass spectrometry analyses. Asterisks indicate significant differences between sF-3 (supernatant of the fungal culture at 3 days) and the corresponding noninoculated control (sC-3). 
the cisZ-type CK, which prevail in maize leaves (Gajdosova et al. 2011; Veach et al. 2003). These CK were largely overlooked and not recorded in most previous reports. Furthermore, our assays focused on local infection sites, which may also explain the contrasting results. It was suggested that most of the obligate and hemibiotrophs analyzed were able to transform transzeatin- $O$-glucoside ( $\mathrm{ZOG}$ ) to the active compound $\mathrm{Z}$, whereas most of the necrotrophs were not (Ashby 2000). This led to the hypothesis that corresponding enzymes secreted by the pathogen at the infection site may convert biologically inactive $\mathrm{CK}$ stored by the host to bioactive CK that, in turn, would stimulate the supply of carbohydrates to the infection sites harboring the pathogen. Our finding that the cisZOG level decreased at the infection site reinforces this hypothesis. The conversion of cisZOG does not necessarily result from fungal enzymatic activity but could also be mediated by a host enzyme induced by infection. Therefore, it is important to note that the CK measurements using fungal in vitro cultures showed that C. graminicola does, indeed, have the capacity to transform cisZOG to cisZ. However, cisZ remained at relatively steady-state levels at infection sites despite the conversion of cisZOG. In this context, it needs to be considered that, in infected leaves, CK levels are influenced by de novo synthesis, import and export from other organs, interconversion, transient and terminal inactivation, as well as perception and signal transduction, all of which could theoretically be mediated by both the host and the pathogen. Because terminal and irreversible inactivation is catalyzed by $\mathrm{CK}$ oxidase/dehydrogenase (CKX), we attempted to determine its activity in extracts obtained from excised tissue samples of green islands. However, CKX activity was undetectable in this limited amount of material using a highly sensitive radioisotope assay with tritiated CK as substrates (data not shown). Furthermore, several findings indicate that not only the free bases, which exhibited relatively constant levels at infection sites in our experiments, have biological activity as typically reported, but also other forms such as CK ribosides (Dolezal et al. 2007; Gajdosova et al. 2011; Holub et al. 1998).

Our results underline the possibility that the cisZ-type CK may play an as-yet not-well-understood role in the interaction between fungi with their hosts. This hypothesis is supported by a recent study on CK levels in the roots of Arabidopsis thaliana after colonization with the mycorrhizal basidiomycete $P i$ riformospora indica (Vadassery et al. 2008). Major changes in concentrations were observed only for the cis isomers, especially for cisZR, which also exhibited the highest level of any CK after infection, as well as for cisZROG and cisZRMP. Although the cisZ concentration appeared somewhat increased in infected roots, compared with the other substances, it remained at relatively low levels (Vadassery et al. 2008). In another study supporting our findings, several CK were analyzed in the pathogenic interaction between the basidiomycete Ustilago maydis and maize (Bruce et al. 2011). Because some of the $\mathrm{CK}$ that we found at higher concentration in maize (e.g., cisZOG) were not resolved in that study, we can only partially compare the results. In $U$. maydis, major increases were observed for the concentrations of cisZR, cisZRMP, $N^{6}-\left(\Delta^{2}\right.$-isopentenyl)adenine-9-riboside- $5^{\prime}$-monophosphate (iPRMP), and cisZ at a later stage of infection, whereas the trans isomers were not affected. Therefore, despite the different types of interactions and the different taxonomic positions of the plant and fungal partners involved, the most prominent effects in cisZ-type CK were similar. However, both the study on $P$. indica (Vadassery et al. 2008) and ours on C. graminicola found higher bioactivity for the trans isomers in several assays. It is interesting to note that $C$. graminicola converts in vitro cisZ to $\mathrm{Z}$ but does not catalyze the reverse reaction. In maize, three
CK receptors have been reported and two of them have a higher affinity for cisZ than for Z (Lomin et al. 2011). It remains open whether both isomers may have specific and distinct functions in planta.

Our feeding experiments using individual CK uncovered a range of additional $\mathrm{CK}$ conversions that the fungus is able to perform in vitro. In addition to the isomerization of cis $\mathrm{Z}$, most notable are the conversions of ZR to cisZRMP, cisZR to ZRMP, and ZRMP to ZR and Z. Based on the data obtained in this study, we propose a model for CK metabolism of $C$. graminicola (Fig. 7). In this model, we have included only reactions supported by our experimental data but did not exclude the possibility that additional conversions may also occur. We infer only reactions leading directly from the respective exogenously amended substances to the experimentally observed compounds, except the supposed conversion of cisZRMP to ZRMP. This hypothesized isomerization explains why the addition of cisZR leads to the appearance of considerable amounts of ZRMP. Alternatively, longer reaction sequences explaining this result would be cisZR $\rightarrow \mathrm{ZR} \rightarrow \mathrm{ZRMP}$ or cis $\mathrm{ZR} \rightarrow$ cis $\mathrm{Z}$ $\rightarrow \mathrm{Z} \rightarrow \mathrm{ZR} \rightarrow \mathrm{ZRMP}$. A central position within the model is taken by ZR that appears to be transformable to several other $\mathrm{CK}$ and that even can be synthesized by the fungus (discussed below). The conversions proposed by our model have been shown to occur in plants (Sakakibara 2006), suggesting that a fungal plant pathogen performs a rather complex CK metabolism that adopts some features also seen in plants.

Beyond the ability to interconvert CK, C. graminicola also has some capacity for $\mathrm{CK}$ biosynthesis. The addition of DMAPP, with AMP, ADP, and ATP, to minimal medium led to synthesis of considerable amounts of a series of CK (i.e., ZRMP, ZR, and Z), indicating that isopentenyltransferase (IPT) activity exists in this fungus. In plants, DMAPP is a substrate for IPT that catalyze the first step in the synthesis of isoprenoid CK. Although, in certain plant-pathogenic fungi, CK biosynthesis has been proposed (Ashby 2000), IPT activities have not been formally proven. In in vitro growing mycelium of $P$. indica, mainly $N^{6}$-( $\Delta^{2}$-isopentenyl)adenine (iP), cisZRMP, and cisZR were detected, and lower amounts of $N^{6}-\left(\Delta^{2}\right.$-isopentenyl)adenine-9-riboside (iPR), cisZROG, and cisZ (Vadassery et al. 2008). Likewise, $U$. maydis produced considerable amounts of cisZRMP and cisZR as well as iPRMP and iPR in vitro (Bruce et al. 2011). Because both studies used rich media containing yeast extract, casein hydrolysate, or peptone, it is unclear whether a complete de novo synthesis of CK occurred or whether these fungi utilized supplemented compounds to generate them. Our results suggest that, during the infection process, $C$. graminicola may not only manipulate CK metabolism in the host by interconverting preexisting CK but also utilize DMAPP to synthesize CK.

In summary, our study provides a comprehensive view of fungal CK metabolism in a fungus-plant pathosystem. Using high spatial and analytical resolution, we have shown that, during infection, fungal CK metabolism is interconnected with that of the host, which adds an additional layer of complexity to the interaction between a hemibiotroph and its host plant. Future work needs to elucidate the contribution of individual $\mathrm{CK}$ to the establishment of a successful infection.

\section{MATERIALS AND METHODS}

\section{Plant and fungal cultivation.}

Maize (Z. mays 'Mikado') (KWS Saat AG, Einbeck, Germany) and wild-type strain $C$. graminicola (Ces.) G. W. Wilson M2 were used for this study. Conditions for cultivation of the fungus and maize in the greenhouse as well as in environmentally controlled growth chambers had been described (Behr et 
al. 2010). For inoculation of 28-day-old maize plants with $C$. graminicola, conidia were sprayed onto the entire plant at a concentration of $1 \times 10^{6} \mathrm{ml}^{-1}$, as reported (Behr et al. 2010).

Phenotypical effects of $\mathrm{CK}$ on senescence (Fig. 1A) were tested by applying appropriate substances $(10 \mu \mathrm{l}$ each) diluted in water onto the surface of detached number 5 leaves of 28 day-old plants. Photographs were taken after 3 days of incubation on wet filter paper in a glass petri dish at ambient temperature and light conditions.

The effects of individual $\mathrm{CK}$ on photosynthesis and transcript levels of maize genes (Fig. 1B and C) were assessed by spraying the substances $(6 \mathrm{ml}$ each) onto whole 28-day-old plants incubated in an environmentally controlled growth chamber under conditions described previously (Behr et al. 2010).

To evaluate the capability of the fungus to convert CK naturally occurring in maize in vitro (Fig. 3), a preculture was established by inoculation of $50 \mathrm{ml}$ of complete medium (Krijger et al. 2008) with $1 \times 10^{6}$ of conidia. The mycelium obtained after $24 \mathrm{~h}$ of growth at $23^{\circ} \mathrm{C}$ and $50 \mathrm{rpm}$ was transferred to $50 \mathrm{ml}$ of sterile distilled water, incubated for $150 \mathrm{~min}$ as before, then transferred to maize leaf extract medium. The latter was prepared from 28-day-old plants by cutting five green or senescing leaves into small pieces, which were subsequently autoclaved in $50 \mathrm{ml}$ of sterile distilled water for 20 min at $121^{\circ} \mathrm{C}$. The resulting supernatant was filter sterilized through a $0.2-\mu \mathrm{m}$ filter and transferred to a $200-\mathrm{ml}$ Erlenmeyer flask. Incubation of the culture continued under the same conditions as described above for 7 days. The mycelium was then harvested on filter paper and the spent medium was filter sterilized through a $0.2-\mu \mathrm{m}$ filter and stored at $-20^{\circ} \mathrm{C}$.

To assess the capability of the fungus to convert commercially available $\mathrm{CK}$ or to utilize precursors for $\mathrm{CK}$ synthesis in vitro (Figs. 4 to 6), $50 \mathrm{ml}$ of Czapek-Dox minimal-medium $\left(0.2 \% \mathrm{NaNO}_{3}, 0.1 \% \mathrm{~K}_{2} \mathrm{HPO}_{4}, 0.05 \% \mathrm{MgSO}_{4} \times 7 \mathrm{H}_{2} \mathrm{O}, 0.05 \%\right.$ $\mathrm{KCl}, 0.001 \% \mathrm{FeSO}_{4}$, and $5 \mathrm{mM}$ Sucrose) in 200-ml Erlenmeyer flasks was inoculated with $1 \times 10^{6}$ conidia. The medium was amended with either individual CK (OlChemIm Ltd., Olomouc, Czech Republic) or with a mixture of putative substrates of isopentenyltransferases, which are involved in CK biosynthesis (i.e., DMAPP, AMP, ADP, and ATP) (all Sigma-Aldrich Chemie $\mathrm{GmbH}$, Taufkirchen, Germany), each at a concentration of $100 \mathrm{nM}$. Incubation was at $23^{\circ} \mathrm{C}$ and $50 \mathrm{rpm}$. Harvest proceeded as given above.

\section{PAM chlorophyll fluorometry and SPAD analyses.}

The impact of CK treatment on photosynthesis and chlorophyll contents in maize leaves was assessed nondestructively by PAM chlorophyll fluorometry and SPAD analysis, respectively. For both, whole plants were treated and incubated as outlined above. Each day, three plants were taken from the growth chamber and PAM and SPAD analyses were performed on 10 randomly chosen areas in the middle of leaf number 3 . For each variant, all 10 measurements were used to calculate

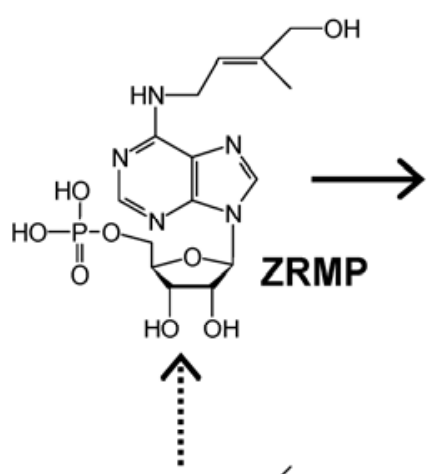

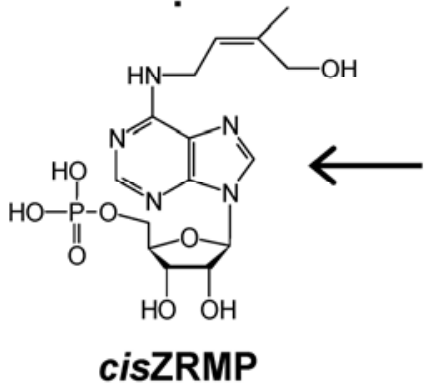

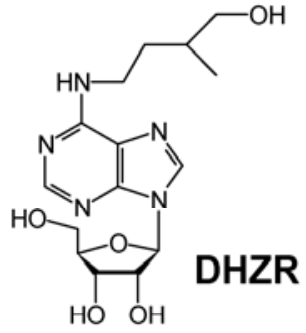

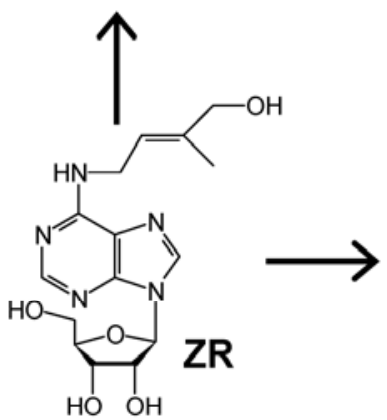<smiles>CCCC</smiles>

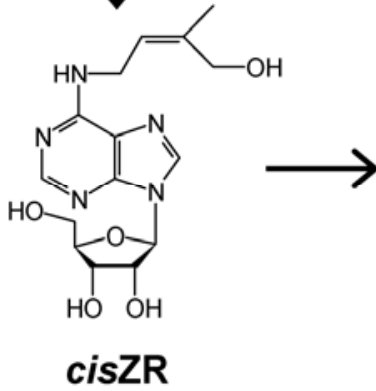<smiles>[Y]Nc1cc(NC/C=C(/C)CO)ncn1</smiles><smiles>CCCC</smiles><smiles>CC(C)(C)C</smiles><smiles>C/C(=C/CNc1ncnc2[nH]cnc12)COC1OC(O)C(O)C1O</smiles>

cisZ

\section{cisZOG}

Fig. 7. Proposed model illustrating cytokinin (CK) conversions by Colletotrichum graminicola in vitro. Straight arrows indicate reactions deduced from data. The broken arrow indicates a presumed conversion to explain the emergence of trans-zeatin-9-riboside-5'-monophosphate (ZRMP) after feeding of ciszeatin-9-riboside (cisZR). 
arithmetic means and standard deviations. We used two handheld devices: a MINI-PAM Photosynthesis Yield Analyzer (Heinz Walz GmbH, Effeltrich, Germany) and a SPAD-502 Chlorophyll Meter (Konica Minolta Sensing Europe B.V., München, Germany). The Mini-PAM instrument assesses the effective quantum yield of photochemical energy conversion in photosynthesis by irradiation with pulse-modulated light and measuring of the chlorophyll fluorescence. The SPAD-502 instrument measures the ratio of the transmitted light at $920 \mathrm{~nm}$, which is not absorbed by chlorophyll, to that at $650 \mathrm{~nm}$, which is absorbed. This ratio is used as a measure of the chlorophyll content of the leaf.

\section{Isolation of RNA and qRT-PCR.}

Four days after spraying of 28-day-old whole maize plants with $100 \mu \mathrm{M} \mathrm{Z}$ or cisZ, leaves were harvested $10 \pm 1 \mathrm{~h}$ after beginning of the light period, immediately frozen in liquid nitrogen, and stored at $-80^{\circ} \mathrm{C}$ until RNA preparation was done as reported (Krijger et al. 2008). For each variant analyzed, RNA was extracted from three pools, each comprising whole leaves (numbers 3 and 5, respectively) from six plants. These pools originated from independent experiments performed on successive weeks. Variants representing mock-inoculated controls were also included. qRT-PCR followed published protocols and primers (Behr et al. 2010), except that the Power SYBR Green RNA-to-CT 1-step kit (Applied Biosystems $\mathrm{GmbH}$ ) was used.

\section{CK determination.}

To measure CK in infected plants at a high spatial resolution (Fig. 2), hundreds of tissue samples carrying green islands on leaf number 3 were excised at 4 dpi with a cannula $(1 \mathrm{~mm}$ in diameter, custom produced at the Fraunhofer Institute for Mechanics of Materials, Polymer Applications, Biological Materials and Interfaces, Halle, Germany), pooled, and frozen in liquid nitrogen. Tissues that surrounded and excluded the green islands were excised with a cork borer ( $5 \mathrm{~mm}$ in diameter).

The analysis of profiles and concentrations of individual CK in both tissue samples (including fungal mycelia) and culture media was performed by HPLC MS/MS. The deeply frozen tissues (equivalent to 0.62 to $2.71 \mathrm{~g}$ of fresh weight [FW] for plant tissue and 0.02 to $0.2 \mathrm{~g}$ of $\mathrm{FW}$ for fungal mycelium) were homogenized in liquid nitrogen, extracted overnight with $10 \mathrm{ml}$ of methanol/water/formic acid (15:4:1 [vol/vol/vol], $\mathrm{pH}$ $2.5,-20^{\circ} \mathrm{C}$ ), and purified by dual-mode solid-phase extraction (Dobrev and Kaminek 2002). The deeply frozen media (equivalent to 1 or $2 \mathrm{ml}$ ) were thawed, acidified (after thorough shaking) to $\mathrm{pH} 2.5$ by formic acid (98\% [ $\mathrm{vol} / \mathrm{vol}])$, and subjected to the same purification procedure as mentioned above using Sep-Pak Plus tC18 cartridges (Waters Corp., Milford, MA, U.S.A.) and Oasis MCX 6- $\mathrm{cm}^{3}$ (150 mg) extraction cartridges (cation exchange and reverse-phase) (Waters Corp.). Separated CK nucleotides were determined as corresponding nucleosides following their dephosphorylation by calf-intestine alkaline phosphatase (Sigma-Aldrich Chemie $\mathrm{GmbH}$ ).

Detection and quantitation of CK employed a HPLC MS/MS system consisting of an HTS-Pal auto-sampler with a cooled sample stack (CTC Analytics AG, Zwingen Switzerland), a quaternary HPLC pump Rheos 2200 (Flux Instruments AG, Reinach, Switzerland), a DeltaChrom CTC 100 column oven (Watrex Prague Ltd., Prague), and a TSQ Quantum Ultra AM triple-quadrupole high-resolution mass spectrometer (Thermo Electron Corp., San Jose, CA, U.S.A.) equipped with an electrospray interface. Dried extracts were redissolved in $100 \mu \mathrm{l}$ of diluted acetonitrile (10\% in water) and filtered by $0.2-\mu \mathrm{m}$ Micro-Spin filters (W. R. Grace \& Co., Columbia, MD, U.S.A.). A $5-\mu l$ aliquot was injected on a $\mathrm{C} 18$
HPLC column Synergy Hydro-RP, 250 by $2 \mathrm{~mm}, 4 \mu \mathrm{m}$ (Phenomenex Inc., Torrance, CA, U.S.A.) and analyzed using a ternary gradient elution (water/acetonitrile $/ 0.01 \%$ acetic acid) starting at $8 \%$ of acetonitrile. The concentration of acetonitrile was increased after $5 \mathrm{~min}$ to $15 \%$ during $10 \mathrm{~min}$ and then to $50 \%$ during $11 \mathrm{~min}$. The portion of $0.01 \%$ acetic acid was maintained at $35 \%$ throughout the analysis. The remainder of the sample was removed from the column by increasing the concentration of acetonitrile to $90 \%$ for $9 \mathrm{~min}$. The column was equilibrated with $8 \%$ acetonitrile for $20 \mathrm{~min}$ before the next sample was injected. The mass spectrometer was operated in the MS/MS mode (selected reaction monitoring) with monitoring of two to four transitions for each compound. The most intensive ion was used for quantification, the others for identity confirmation. CK were quantified by the Xcalibur software (version 2.0; Thermo Fisher Scientific Inc., San Jose, CA, U.S.A.) using multilevel internal standards calibration graphs with $\left[{ }^{2} \mathrm{H}\right]$-labeled CK used as internal standards (OlChemIm Ltd.). The quantitative data were expressed as picomoles per sample. The calibration allowed for quantification at a range of 0.1 to $500 \mathrm{pmol} / \mathrm{sample}$. In total, 26 different CK were analyzed, although concentrations of some of them were below the detection limit. CK were abbreviated as proposed earlier (Kaminek et al. 2000). Statistical analyses employed the unpaired $t$ test; differences at $P<0.05$ were considered significant.

\section{ACKNOWLEDGMENTS}

We thank M. Korecká for excellent technical assistance, E. Vollmer for skillful greenhouse services, and J.-J. Krijger for helpful discussions. This work was supported by a Deutsche Forschungsgemeinschaft (DFG) grant provided in the framework of the SFB 648 (project A5, to S. G. R. Wirsel and H. B. Deising) and a grant from the State of Sachsen-Anhalt provided in the framework of the Interdisciplinary Center for Crop Plant Research (Interdisziplinäres Zentrum für Nutzpflanzenforschung; IZN, project 08 , to S. G. R. Wirsel), the Czech Science Foundation (P506/11/0774, to V. Motyka), and the Ministry of Education, Youth and Sports of the Czech Republic (LC06034, to J. Malbeck and V. Motyka).

\section{LITERATURE CITED}

Ashby, A. M. 2000. Biotrophy and the cytokinin conundrum. Physiol. Mol. Plant Pathol. 57:147-158.

Balibrea Lara, M. E., Gonzales Garcia, M.-C., Fatima, T., Ehness, R., Lee, T. K., Proels, R., Tanner, W., and Roitsch, T. 2004. Extracellular invertase is an essential component of cytokinin-mediated delay of senescence. Plant Cell 16:1276-1287.

Barker, S. J., and Tagu, D. 2000. The roles of auxins and cytokinins in mycorrhizal symbioses. J. Plant Growth Regul. 19:144-154.

Bassil, N. V., Mok, D. W. S., and Mok, M. C. 1993. Partial purification of a cis-trans-isomerase of zeatin from immature seed of Phaseolus vulgaris L. Plant Physiol. 102:867-872.

Behr, M., Humbeck, K., Hause, G., Deising, H. B., and Wirsel, S. G. R. 2010. The hemibiotroph Colletotrichum graminicola locally induces photosynthetically active green islands but globally accelerates senescence on aging maize leaves. Mol. Plant-Microbe Interact. 23:879-892.

Bruce, S. A., Saville, B. J., and Emery, R. J. N. 2011. Ustilago maydis produces cytokinins and abscisic acid for potential regulation of tumor formation in maize. J. Plant Growth Regul. 30:51-63.

Buchanan-Wollaston, V. 1997. The molecular biology of leaf senescence. J. Exp. Bot. 48:181-199.

Costacurta, A., and Vanderleyden, J. 1995. Synthesis of phytohormones by plant-associated bacteria. Crit. Rev. Microbiol. 21:1-18.

Dobrev, P. I., and Kaminek, M. 2002. Fast and efficient separation of cytokinins from auxin and abscisic acid and their purification using mixedmode solid-phase extraction. J. Chromatogr. A 950:21-29.

Dolezal, K., Popa, I., Hauserova, E., Spichal, L., Chakrabarty, K., Novak, O., Krystof, V., Voller, J., Holub, J., and Strnad, M. 2007. Preparation, biological activity and endogenous occurrence of N-6-benzyladenosines. Bioorgan. Med. Chem. 15:3737-3747.

Frebort, I., Kowalska, M., Hluska, T., Frebortova, J., and Galuszka, P. 2011. Evolution of cytokinin biosynthesis and degradation. J. Exp. Bot. 62:2431-2452.

Frugier, F., Kosuta, S., Murray, J. D., Crespi, M., and Szczyglowski, K. 
2008. Cytokinin: Secret agent of symbiosis. Trends Plant Sci. 13:115120

Gajdosova, S., Spichal, L., Kaminek, M., Hoyerova, K., Novak, O., Dobrev, P. I., Galuszka, P., Klima, P., Gaudinova, A., Zizkova, E., Hanus, J., Dancak, M., Travnicek, B., Pesek, B., Krupicka, M., Vankova, R., Strnad, M., and Motyka, V. 2011. Distribution, biological activities, metabolism, and the conceivable function of cis-zeatin-type cytokinins in plants. J. Exp. Bot. 62:2827-2840.

Holub, J., Hanus, J., Hanke, D. E., and Strnad, M. 1998. Biological activity of cytokinins derived from ortho- and meta-hydroxybenzyladenine. Plant Growth Regul. 26:109-115.

Hoyerova, K., Gaudinova, A., Malbeck, J., Dobrev, P. I., Kocabek, T., Solcova, B., Travnickova, A., and Kaminek, M. 2006. Efficiency of different methods of extraction and purification of cytokinins. Phytochemistry 67:1151-1159.

Kakimoto, T. 2001. Identification of plant cytokinin biosynthetic enzymes as dimethylallyl diphosphate : ATP/ADP isopentenyltransferases. Plant Cell Physiol. 42:677-685.

Kaminek, M., Paces, V., Corse, J., and Challice, J. S. 1979. Effect of stereospecific hydroxylation of N6-(delta-2-isopentenyl)adenosine on cytokinin activity. Planta 145:239-243.

Kaminek, M., Brezinova, A., Gaudinova, A., Motyka, V., Vankova, R., and Zazimalova, E. 2000. Purine cytokinins: A proposal of abbreviations. Plant Growth Regul. 32:253-256.

Kasahara, H., Takei, K., Ueda, N., Hishiyama, S., Yamaya, T., Kamiya, Y., Yamaguchi, S., and Sakakibara, H. 2004. Distinct isoprenoid origins of cis- and trans-zeatin biosyntheses in Arabidopsis. J. Biol. Chem. 279:14049-14054.

Kieber, J. J., and Schaller, G. E. 2010. The perception of cytokinin: A story 50 years in the making. Plant Physiol. 154:487-492.

Kim, H. J., Ryu, H., Hong, S. H., Woo, H. R., Lim, P. O., Lee, I. C., Sheen, J., Nam, H. G., and Hwang, I. 2006. Cytokinin-mediated control of leaf longevity by AHK3 through phosphorylation of ARR2 in Arabidopsis. Proc. Natl. Acad. Sci. U.S.A. 103:814-819.

Kojima, M., Kamada-Nobusada, T., Komatsu, H., Takei, K., Kuroha, T., Mizutani, M., Ashikari, M., Ueguchi-Tanaka, M., Matsuoka, M., Suzuki, K., and Sakakibara, H. 2009. Highly sensitive and high-throughput analysis of plant hormones using MS-probe modification and liquid chromatography tandem mass spectrometry: An application for hormone profiling in Oryza sativa. Plant Cell Physiol. 50:1201-1214.

Krijger, J.-J., Horbach, R., Behr, M., Schweizer, P., Deising, H. B., and Wirsel, S. G. R. 2008. The yeast signal sequence trap identifies secreted proteins of the hemibiotrophic corn pathogen Colletotrichum graminicola. Mol. Plant-Microbe Interact. 21:1325-1336.

Kyozuka, J. 2007. Control of shoot and root meristem function by cytokinin. Curr. Opin. Plant Biol. 10:442-446.

Letham, D. S., and Palni, L. M. S. 1983. The biosynthesis and metabolism of cytokinins. Annu. Rev. Plant Physiol. Plant Mol. Biol. 34:163-197.
Lomin, S. N., Yonekura-Sakakibara, K., Romanov, G. A., and Sakakibara, H. 2011. Ligand-binding properties and subcellular localization of maize cytokinin receptors. J. Exp. Bot. 62:5149-5159.

Ludwig-Müller, J., and Schuller, A. 2008. What can we learn from clubroots: Alterations in host roots and hormone homeostasis caused by Plasmodiophora brassicae. Eur. J. Plant Pathol. 121:291-302.

Matsumoto-Kitano, M., Kusumoto, T., Tarkowski, P., Kinoshita-Tsujimura, K., Vaclavikova, K., Miyawaki, K., and Kakimoto, T. 2008. Cytokinins are central regulators of cambial activity. Proc. Natl. Acad. Sci. U.S.A. 105:20027-20031.

Mok, D. W. S., and Mok, M. C. 2001. Cytokinin metabolism and action Annu. Rev. Plant Physiol. Plant Mol. Biol. 52:89-118.

Novak, O., Hauserova, E., Amakorova, P., Dolezal, K. and Strnad, M. 2008. Cytokinin profiling in plant tissues using ultra-performance liquid chromatography-electrospray tandem mass spectrometry. Phytochemistry 69:2214-2224.

Pernisova, M., Klima, P., Horak, J., Valkova, M., Malbeck, J., Soucek, P., Reichman, P., Hoyerova, K., Dubova, J., Friml, J., Zazimalova, E., and Hejatko, J. 2009. Cytokinins modulate auxin-induced organogenesis in plants via regulation of the auxin efflux. Proc. Natl. Acad. Sci. U.S.A. 106:3609-3614.

Pertry, I., Vaclavikova, K., Gemrotova, M., Spíchal, L., Galuszka, P., Depuydt, S., Temmerman, W., Stes, E., De Keyser, A., Riefler, M. Biondi, S., Novák, O., Schmülling, T., Strnad, M., Tarkowski, P., Holsters, M., and Vereecke, D. 2010. Rhodococcus fascians impacts plant development through the dynamic Fas-mediated production of a cytokinin mix. Mol. Plant-Microbe Interact. 23:1164-1174.

Sakakibara, H. 2006. Cytokinins: Activity, biosynthesis, and translocation. Annu. Rev. Plant Biol. 57:431-449.

Sakakibara, H., Kasahara, H., Ueda, N., Kojima, M., Takei, K., Hishiyama, S., Asami, T., Okada, K., Kamiya, Y., Yamaya, T., and Yamaguchi, S. 2005. Agrobacterium tumefaciens increases cytokinin production in plastids by modifying the biosynthetic pathway in the host plant. Proc. Natl. Acad. Sci. U.S.A. 102:9972-9977.

Vadassery, J., Ritter, C., Venus, Y., Camehl, I., Varma, A., Shahollari, B. Novak, O., Strnad, M., Ludwig-Müller, J., and Oelmüller, R. 2008. The role of auxins and cytokinins in the mutualistic interaction between Arabidopsis and Piriformospora indica. Mol. Plant-Microbe Interact. 21:1371-1383.

Veach, Y. K., Martin, R. C., Mok, D. W., Malbeck, J., Vankova, R., and Mok, M. C. 2003. O-glucosylation of cis-zeatin in maize. Characterization of genes, enzymes, and endogenous cytokinins. Plant Physiol. 131:1374-1380.

Walters, D. R., and McRoberts, N. 2006. Plants and biotrophs: A pivotal role for cytokinins? Trends Plant Sci. 11:581-586.

Wingler, A., and Roitsch, T. 2008. Metabolic regulation of leaf senescence: Interactions of sugar signalling with biotic and abiotic stress responses. Plant Biol. 10:50-62. 